\title{
Parametrization-free determination of the shape parameters for the pion electromagnetic form factor
}

\author{
B. Ananthanarayan ${ }^{a}$, I. Caprini ${ }^{b}{\text {, Diganta } \text { Das }^{c} \text {, and I. Sentitemsu Imsong }}^{d}$ \\ ${ }^{a}$ Centre for High Energy Physics, Indian Institute of Science, Bangalore 560 012, India \\ ${ }^{b}$ Horia Hulubei National Institute for Physics and Nuclear Engineering, P.O.B.MG-6, 077125 Bucharest-Magurele, Romania \\ ${ }^{c}$ Institute of Mathematical Sciences, Taramani, Chennai 600113, India \\ ${ }^{d}$ Theoretische Physik 1, Naturwissenschaftlich-Technische Fakultät, Universität Siegen, D-57068 Siegen, Germany
}

the date of receipt and acceptance should be inserted later

\begin{abstract}
Recent data from high statistics experiments that have measured the modulus of the pion electromagnetic form factor from threshold to relatively high energies are used as input in a suitable mathematical framework of analytic continuation to find stringent constraints on the shape parameters of the form factor at $t=0$. The method uses also as input a precise description of the phase of the form factor in the elastic region based on Fermi-Watson theorem and the analysis of the $\pi \pi$ scattering amplitude with dispersive Roy equations, and some information on the spacelike region coming from recent high precision experiments. Our analysis confirms the inconsistencies of several data on the modulus, especially from low energies, with analyticity and the input phase, noted in our earlier work. Using the data on the modulus from energies above $0.65 \mathrm{GeV}$, we obtain, with no specific parametrization, the prediction $\left\langle r_{\pi}^{2}\right\rangle \in(0.42,0.44) \mathrm{fm}^{2}$ for the charge radius. The same formalism leads also to very narrow allowed ranges for the higher-order shape parameters at $t=0$, with a strong correlation among them.
\end{abstract}

PACS. 11.55.Fv, 13.40.Gp, 25.80.Dj

\section{Introduction}

The pion electromagnetic form factor is an important quantity that encodes information on the structure of the strong interactions. At high spacelike momenta, it acts as an excellent testing ground for studying the onset of perturbative QCD for exclusive quantities, while at low energies it is an important laboratory for the study of chiral symmetry breaking. It plays a major role in the precision tests of the Standard Model, through its contribution to the anomalous magnetic moment of the muon, which is one of the most precisely measured observables in particle physics.

The derivatives of the form factor at $t=0$ present in the Taylor series expansion

$$
F(t)=1+\frac{1}{6}\left\langle r_{\pi}^{2}\right\rangle t+c t^{2}+d t^{3}+\cdots
$$

are quantities of interest for testing the expansions of chiral perturbation theory (ChPT) and the lattice calculations of the pion form factor. These shape parameters, especially the charge radius squared $\left\langle r_{\pi}^{2}\right\rangle$, are the object of many investigations. However, the point $t=0$ is not directly accessible to experiment. Measurements near the origin [1]-[5] in the spacelike region $t<0$ have been exploited in the past to yield the charge radius and the higher shape parameters. Recent high statistics experiments [6]-11] have measured the modulus in the timelike region on the unitarity cut. Since this experimental information is available only quite far away from the origin, suitable techniques of extrapolation have to be invented in order to obtain a reliable extraction of the shape parameters from these data.

The analyticity properties of the form factor are very useful in performing this extrapolation. From causality and unitarity it is known that $F(t)$ is an analytic function in the complex $t$-plane, with a cut starting at the unitarity threshold $t_{+}=4 M_{\pi}^{2}$ and running to infinity. The FermiWatson theorem relates the phase of the form factor in the elastic region, $t_{+} \leq t \leq t_{\text {in }}$, where $t_{\text {in }}$ is the first inelastic threshold, to the $P$-wave phase shift of $\pi \pi$ scattering, which is now known to high precision due to studies based on Roy equations for the $\pi \pi$ elastic amplitude [12]-[16].

Analyticity was exploited in the past by means of various types of dispersive representations, which express the function in terms of either the imaginary part, the phase or the modulus on the cut. However, none of the standard dispersion relations has complete input, and their application requires ad-hoc assumptions. Specific parametrizations of the data were also frequently used, but they are affected by the known "instability" of analytic continu- 
ation [17] if they are extrapolated outside their original range of validity.

Recently, more sophisticated methods of complex analysis were applied in a formalism known as "theory of unitarity bounds" (for a review and earlier references see [18]). The main feature of this approach is that it allows the optimal implementation of the phase and modulus information available on the unitarity cut. Using mathematical techniques belonging to the analytic interpolation theory on Hardy spaces, one can include as input also values of the function or its derivatives at discrete points inside the analyticity domain. This input is shown to lead to strong constrains on other values of the form factor, like the shape parameters at the origin or the values on the spacelike axis.

The inherent limitation of this approach is that it produces only bounds on the quantities of interest, rather than definite determinations. Furthermore, when the experimental errors are large, there is no significant advantage in adding more and more input information, as the bounds do not improve. On the other hand, due to the increased accuracy of the input observed recently, the bounds can be very stringent, competing in precision with experimental data or theoretical predictions.

Applications of this formalism to the pion form factor were undertaken in [19] and more recently in [20, 21, 22]. In [20], the phase in the elastic region $t<t_{\text {in }}$, where $t_{\text {in }}$ is the first important inelastic threshold set by the production of an $\omega \pi$ pair, together with the modulus above $t_{\text {in }}$ used in an averaged way and some spacelike information, were exploited for deriving constraints on the higher shape parameters $c$ and $d$ appearing in the expansion (10).

In a more recent work [22], the same input was used for deriving bounds on the modulus in the elastic region, below the inelastic threshold. The results provided nontrivial consistency checks on the recent experimental data [6]-[11] on the modulus from $e^{+} e^{-}$annihilation and $\tau$-decay experiments. In particular, at low energies the calculated bounds offered a more precise description of the modulus than the experimental data.

In 20, 21, 22], the experimental data on the modulus below the inelastic threshold were not included as input. The results showed that the knowledge of the phase in the elastic region has strong implications for constraining the shape of the form factor below and above the unitarity threshold. The inclusion of the experimental measurements on the modulus of the form factor below the inelastic threshold is the object of the present work. More precisely, we ask what is the import of these measurements on the radius $\left\langle r_{\pi}^{2}\right\rangle$ and the higher derivatives $c$ and $d$ at $t=0$. Thus, the present work extends our previous analysis of the shape parameters in [20], where the information on the modulus below $t_{\text {in }}$ was not used. It complements the work done in Refs. [20, 21, 22] and provides a further consistency check on the experimental data.

There is a rich discussion in the literature on the determination of the shape parameters. On the theoretical side, a fit based on ChPT to two-loop accuracy for $\tau$ decays gives $\left\langle r_{\pi}^{2}\right\rangle=(0.431 \pm 0.020 \pm 0.016) \mathrm{fm}^{2}$ and $c=\left(3.2 \pm 0.5_{\exp } \pm 0.9_{\text {theor }}\right) \mathrm{GeV}^{-4}[23]$. The pion form factor has been calculated in two-loop ChPT which gives $\left\langle r_{\pi}^{2}\right\rangle=(0.437 \pm 0.016) \mathrm{fm}^{2}$ and $c=(3.85 \pm 0.60) \mathrm{GeV}^{-4}$ [24]. In [25], the values $\left\langle r_{\pi}^{2}\right\rangle=(0.452 \pm 0.013) \mathrm{fm}^{2}$ and $c=(4.49 \pm 0.28) \mathrm{GeV}^{-4}$ were obtained within the three flavour framework and at next-to-next-to-leading order in ChPT. A theoretical determination based on quark Dyson-Schwinger equation combined with Bethe-Salpeter equation for meson amplitudes yields $\left\langle r_{\pi}^{2}\right\rangle=0.45 \mathrm{fm}^{2}[26$ ]. Recent lattice calculations with chiral extrapolation based on two-loop ChPT give $\left\langle r_{\pi}^{2}\right\rangle=0.409(23)(37) \mathrm{fm}^{2}$ and $c=$ $3.22(17)(36) \mathrm{GeV}^{-4}$ [27]. Phase (Omnès-type) representations with various parametrizations of the phase along the whole unitarity cut give $\left\langle r_{\pi}^{2}\right\rangle=(0.432 \pm 0.001) \mathrm{fm}^{2}$ and $\left.c=(3.84 \pm 0.02) \mathrm{GeV}^{-4} 28\right]$. Recently, a fit of spacelike data with Padé approximants [29] gave the values $\left\langle r^{2}\right\rangle=$ $(0.445 \pm 0.002 \pm 0.007) \mathrm{fm}^{2}$ and $c=(3.30 \pm 0.03 \pm 0.33)$ $\mathrm{GeV}^{-4}$

Other values are $\left\langle r_{\pi}^{2}\right\rangle=(0.427 \pm 0.010) \mathrm{fm}^{2}$, quoted in [1], and $\left\langle r_{\pi}^{2}\right\rangle^{\frac{1}{2}}=(0.711 \pm 0.009) \mathrm{fm}$ given in [3], both being obtained by fits of the data with a simple pole. The curvature $c$ has also been determined from fits of experimental data with specific analytic parametrizations of the form factor. The value $c=(3.90 \pm 0.10) \mathrm{GeV}^{-4}$ has been obtained in 30] by a standard dispersion relation. A fit of the ALEPH data [31] on the hadronic $\tau$ decay rate with a Gounaris-Sakurai formula for the form factor 32 gives $c=(3.2 \pm 1.0) \mathrm{GeV}^{-4}$. The analysis based on the technique of unitarity bounds [20] mentioned above led to the range $3.75 \mathrm{GeV}^{-4} \lesssim c \lesssim 3.98 \mathrm{GeV}^{-4}$.

The next shape parameter $d$ is much less well known. Theoretical results from ChPT and lattice calculations are not yet available. The value $d=(9.70 \pm 0.40) \mathrm{GeV}^{-6}$ has been obtained from fits of data by means of usual dispersion relations [30], while the Taylor expansion of the Gounaris-Sakurai parametrization [31], leads to $d=$ $9.80 \mathrm{GeV}^{-6}$. In [20] we derived the range $9.91 \mathrm{GeV}^{-6} \lesssim$ $d \lesssim 10.46 \mathrm{GeV}^{-6}$.

Except for the ranges predicted in 20], the previous determinations of the shape parameters came from specific parametrizations, like polynomial expansions, Padé approximants or the Gounaris-Sakurai model for the dominant $\rho$ pole. In contrast, the predictions of the present work are parametrization-free in the sense that we do not rely on specific analytic expressions of the form factor or its modulus. The determination follows from general principles of analyticity, with an input consisting from the phase in the elastic region and a conservative condition on the modulus on the unitarity cut. The method also avoids the instability problems inherent in the analytic continuation of specific parametrizations.

The scheme of the paper is as follows: in Sec. 2] we present very briefly the mathematical formalism whereas in Sec. 3 we describe the information used as input to our work. In Sec. 4, we present the results of our investigations. In subsection 4.1 we present the analysis of the charge radius $\left\langle r_{\pi}^{2}\right\rangle$ and in subsection 4.2 the analysis of the higher shape parameters $c$ and $d$. In Sec. [5] we summarize our results and present our conclusions. 


\section{Method}

We use the Fermi-Watson theorem, which states that

$$
\operatorname{Arg}[F(t+i \epsilon)]=\delta_{1}^{1}(t), \quad t_{+} \leq t \leq t_{\mathrm{in}},
$$

where $\delta_{1}^{1}(t)$ is the phase-shift of the $P$-wave of $\pi \pi$ elastic scattering and $t_{\text {in }}$ the first inelastic threshold. In addition, we exploit the recent experimental data on the modulus above $t_{\text {in }}$ and the $1 / t$ asymptotic decrease of the form factor predicted by perturbative QCD, by adopting the relation

$$
\frac{1}{\pi} \int_{t_{\text {in }}}^{\infty} d t \rho(t)|F(t)|^{2}=I
$$

where $\rho(t)$ is a suitable positive-definite weight allowing a reliable calculation of the value of $I$. As in [20, 21, 22] we consider weights of the form

$$
\rho(t)=\frac{t^{\beta}}{\left(t+Q^{2}\right)^{\gamma}},
$$

where $Q^{2} \geq 0$ and $\beta, \gamma$ are taken in the range $\beta \leq \gamma \leq$ $\beta+2$.

The conditions (2) and (3) can be written in a form that allows the application of the mathematical interpolation theory for analytic functions [33, 34]. We first exploit (2) by introducing the Omnès function

$$
\mathcal{O}(t)=\exp \left(\frac{t}{\pi} \int_{t_{+}}^{\infty} d t^{\prime} \frac{\delta\left(t^{\prime}\right)}{t^{\prime}\left(t^{\prime}-t\right)}\right)
$$

where $\delta(t)=\delta_{1}^{1}(t)$ for $t \leq t_{\mathrm{in}}$, and is an arbitrary function, sufficiently smooth (i.e., Lipschitz continuous) for $t>t_{\text {in }}$. The crucial observation is that the function $h(t)$, defined by

$$
F(t)=\mathcal{O}(t) h(t),
$$

is real for $t \leq t_{\mathrm{in}}$, i.e. it is analytic in the $t$-plane cut only along $t>t_{\text {in }}$. Furthermore, from (3) it follows that $h(t)$ satisfies the condition

$$
\frac{1}{\pi} \int_{t_{\mathrm{in}}}^{\infty} d t \rho(t)|\mathcal{O}(t)|^{2}|h(t)|^{2}=I
$$

This relation can be written in a canonical form by performing the conformal transformation

$$
z \equiv \tilde{z}(t)=\frac{\sqrt{t_{\mathrm{in}}}-\sqrt{t_{\mathrm{in}}-t}}{\sqrt{t_{\mathrm{in}}}+\sqrt{t_{\mathrm{in}}-t}},
$$

which maps the upper (lower) lip of the branch-cut $\left[t_{\text {in }}, \infty\right]$ to the upper (lower) half of the unit circle in the complex $z$-plane, and the cut $t$-plane onto the interior of the unit circle $|z|<1$, the real line $[-\infty, 0]$ to $[-1,0]$ and $\left[0, t_{\mathrm{in}}\right]$ to $[0,1]$. We introduce then two outer functions, i.e. functions analytic and without zeros in the unit disk $|z|<1$, defined in terms of their modulus on the boundary, related to $\sqrt{\rho(t)|\mathrm{d} t / \mathrm{d} \tilde{z}(t)|}$ and $|\mathcal{O}(t)|$, respectively [19, 18]. In particular, for weight functions of the form (4), the first outer function $w(z)$ can be written in an analytic closed form in the $z$-variable as 18

$$
w(z)=\left(2 \sqrt{t_{\mathrm{in}}}\right)^{1+\beta-\gamma} \frac{(1-z)^{1 / 2}}{(1+z)^{3 / 2-\gamma+\beta}} \frac{\left(1+\tilde{z}\left(-Q^{2}\right)\right)^{\gamma}}{\left(1-z \tilde{z}\left(-Q^{2}\right)\right)^{\gamma}} .
$$

For the second outer function, denoted as $\omega(z)$, we use an integral representation in terms of its modulus on the cut $t>t_{\text {in }}$, which can be written as 19,18

$$
\omega(z)=\exp \left(\frac{\sqrt{t_{\text {in }}-\tilde{t}(z)}}{\pi} \int_{t_{\text {in }}}^{\infty} \frac{\ln \left|\mathcal{O}\left(t^{\prime}\right)\right| \mathrm{d} t^{\prime}}{\sqrt{t^{\prime}-t_{\text {in }}}\left(t^{\prime}-\tilde{t}(z)\right)}\right),
$$

where $\tilde{t}(z)$ is the inverse of $z=\tilde{z}(t)$, for $\tilde{z}(t)$ defined in (8).

Further, we define a function $g(z)$ by

$$
g(z)=w(z) \omega(z) F(\tilde{t}(z))[\mathcal{O}(\tilde{t}(z))]^{-1},
$$

such that (7) can be written in terms of the integral of $|g(z)|^{2}$ on the boundary $\left(z=\mathrm{e}^{i \theta}\right)$ as

$$
\frac{1}{2 \pi} \int_{0}^{2 \pi} \mathrm{d} \theta\left|g\left(\mathrm{e}^{i \theta}\right)\right|^{2}=I .
$$

The $L^{2}$-norm equality (12) is the input condition to what is known as the Meiman interpolation problem 33], which consists of finding the most general rigorous correlations between the values of the function and its derivatives inside the unit disk $|z|<1$ consistent with the relation (12) (for a proof and older references see [18]).

For instance, with techniques of complex analysis, one can show that (12) implies the following determinantal inequality :

$$
\left|\begin{array}{ccccc}
\bar{I} & \bar{\xi}_{1} & \bar{\xi}_{2} & \cdots & \bar{\xi}_{N} \\
\bar{\xi}_{1} & \frac{z_{1}^{2 K}}{1-z_{1}^{2}} & \frac{\left(z_{1} z_{2}\right)^{K}}{1-z_{1} z_{2}} & \cdots & \frac{\left(z_{1} z_{N}\right)^{K}}{1-z_{1} z_{N}} \\
\bar{\xi}_{2} & \frac{\left(z_{1} z_{2}\right)^{K}}{1-z_{1} z_{2}} & \frac{\left(z_{2}\right)^{2 K}}{1-z_{2}^{2}} & \cdots & \frac{\left(z_{2} z_{N}\right)^{K}}{1-z_{2} z_{N}} \\
\vdots & \vdots & \vdots & \vdots & \vdots \\
\bar{\xi}_{N} & \frac{\left(z_{1} z_{N}\right)^{K}}{1-z_{1} z_{N}} & \frac{\left(z_{2} z_{N}\right)^{K}}{1-z_{2} z_{N}} & \cdots & \frac{z_{N}^{2 K}}{1-z_{N}^{2}}
\end{array}\right| \geq 0,
$$

where the auxiliary quantities

$$
\bar{I}=I-\sum_{k=0}^{K-1} g_{k}^{2}, \quad \bar{\xi}_{n}=g\left(z_{n}\right)-\sum_{k=0}^{K-1} g_{k} z_{n}^{k}
$$

are defined in terms of the values :

$$
\begin{aligned}
{\left[\frac{1}{k !} \frac{d^{k} g(z)}{d z^{k}}\right]_{z=0} } & =g_{k}, \quad 0 \leq k \leq K-1, \\
g\left(z_{n}\right) & =\xi_{n}, \quad 1 \leq n \leq N .
\end{aligned}
$$

For simplicity we considered $N$ real points $z_{n} \in(-1,1)$ and $K-1$ derivatives at $z=0$. The details of the derivation are reviewed in Ref. [18]. 
By using (11) one can express the inequality (13) as a quadratic constraint on the values of the form factor $F(t)$ and its derivatives at specific points. From this, by solving simple quadratic equations, one can derive upper and lower bounds on one of the first $K$ derivatives at $t=0$, in terms of the other values included in the set. In particular, if the point $t_{n}$ is situated on the elastic part of the cut, i.e. $t_{+}<t_{n}<t_{\text {in }}$, the relation (16) writes as

$$
g\left(z_{n}\right)=w\left(z_{n}\right) \omega\left(z_{n}\right)\left|F\left(t_{n}\right)\right| /\left|\mathcal{O}\left(t_{n}\right)\right|,
$$

where $z_{n}=\tilde{z}\left(t_{n}\right)$ and the modulus $|\mathcal{O}(t)|$ of the Omnès function is obtained from (5) by the Principal Value (PV) Cauchy integral

$$
|\mathcal{O}(t)|=\exp \left(\frac{t}{\pi} \mathrm{PV} \int_{4 M_{\pi}^{2}}^{\infty} d t^{\prime} \frac{\delta\left(t^{\prime}\right)}{t^{\prime}\left(t^{\prime}-t\right)}\right) .
$$

\section{Input}

As mentioned above, the first inelastic threshold $t_{\text {in }}$ for the pion form factor is due to the opening of the $\omega \pi$ channel which corresponds to $\sqrt{t_{\mathrm{in}}}=M_{\omega}+M_{\pi}=0.917 \mathrm{GeV}$. Below $t_{\text {in }}$ Fermi-Watson theorem (2) relates the phase of the form factor to the $P$-wave phase shift $\delta_{1}^{1}(t)$ determined recently with high precision from Roy equations satisfied by the $\pi \pi$ elastic amplitude [12]-[16]. In fact, some input to these equations is borrowed from the knowledge of the pion form factor itself: for instance, the value of the $P$-wave phase shift at the matching point of $0.8 \mathrm{GeV}$ was taken in 12, 13, 14 from a Gounaris-Sakurai parametrization of the CLEO data on the form factor [35]. As discussed in detail in Ref. [22], this input may be improved with the new data on the modulus of the form factor. The purpose of our analyses, reported in [20, 21, 22] and continued here, is precisely to provide analyticity tests leading gradually to a more accurate determination of the form factor at low energies.

We calculate the Omnès function (5) using as input for $t \leq t_{\text {in }}$ the phase shift $\delta_{1}^{1}(t)$ from Refs. [14] and [16], which we denote as Bern and Madrid phase, respectively. Above $t_{\text {in }}$ we use a continuous function $\delta(t)$, which approaches asymptotically $\pi$. As shown in [18], if this function is Lipschitz continuous, the dependence on $\delta(t)$ of the functions $\mathcal{O}(t)$ and $\omega(z)$, defined in (5) and (10), respectively, exactly compensate each other, leading to results fully independent of the unknown phase in the inelastic region. This is one of the important strengths of the method applied in this work.

In our analysis we use the condition (3) with weights of the type (4). We calculate the integral defined in (3) using the BaBar data [6] from $t_{\text {in }}$ up to $\sqrt{t}=3 \mathrm{GeV}$, continued with a constant value for the modulus in the range $3 \mathrm{GeV} \leq \sqrt{t} \leq 20 \mathrm{GeV}$, smoothly connected with a $1 / t$ decrease above $20 \mathrm{GeV}$. As discussed in [22], the choice of the weight should lead to an accurate value for the integral $I$, providing at the same time a strong constraint on the high energy behaviour of the form factor. We have tested a large class of parameters in (4), and found that the results for the shape parameters obtained with various weights are very similar. In our analysis we adopted the weight $\rho(t)=1 / t$, for which the value of $I$ is [22]:

$$
I=0.578 \pm 0.022,
$$

where the uncertainty is due to the BaBar experimental errors. As shown in [19, 18], for a fixed weight the bounds depend in a monotonous way on the value of the quantity $I$, becoming stronger/weaker when this value is decreased/increased. In the applications, we have used as input the central value of $I$ given in (18) increased by the error, which leads to the most conservative bounds.

We use as input also the values at several real points inside the analyticity domain $|z|<1$, which translate as $t<t_{\text {in }}$ in the $t$-plane. Specifically, we implement the normalization condition implemented in the expansion (10):

$$
F(0)=1,
$$

and one of the spacelike data taken from [4, 5]:

$$
\begin{gathered}
F\left(-1.60 \mathrm{GeV}^{2}\right)=0.243 \pm 0.012_{-0.008}^{+0.019} \\
F\left(-2.45 \mathrm{GeV}^{2}\right)=0.167 \pm 0.010_{-0.007}^{+0.013}
\end{gathered}
$$

In addition, we have used the value of the modulus $F\left(t_{n}\right)$ at an energy below the $\omega \pi$ inelastic threshold,

$$
\left|F\left(t_{n}\right)\right|=F_{n} \pm \epsilon_{n}, \quad t_{+}<t_{n}<t_{\text {in }} .
$$

with the central value $F_{n}$ and the error $\epsilon_{n}$ taken from one of the recent experiments [6]-[11].

In our work we implemented the modulus at a single energy and varied this energy over the available experimental range. Of course, the formalism allows the simultaneous inclusion of more points below the inelastic threshold, and in principle the inclusion of each new input leads to stronger constraints. However, we recall that the information used as input at the initial points leads to an allowed domain for the value at each new point. On the other hand, each new input is known within an error channel. It may happen that the allowed and the experimental ranges are disjoint. This would signal an inconsistency in the input data, in which case the mathematical problem with an additional input has no solution. If the experimental range of the additional input is consistent with the allowed range determined by the previous values and is narrower than this range, its inclusion clearly improves the results. However, it turns out that for nonzero experimental errors of the present size, one reaches rather quickly a saturation, i.e., adding one more datum leads to bounds that are no better than the bounds obtained without that additional datum. In practice, as discussed in [20], one stops at a reasonable number $N$ when the gain from including a larger number of points is not significant. Our analysis shows that already with one point taken from the spacelike region and another from the timelike region we obtain rather narrow allowed ranges for the quantities of interest. Thus we stop at $N=2$ and vary the energy 
of the input modulus. The a posteriori comparison of the bounds obtained with modulus from different energies will show that actually there are some inconsistencies between different data.

We mention that we work in the exact isospin limit, taking into account the isospin violation due to the $\rho-$ $\omega$ mixing in $e^{+} e^{-}$annihilation by a standard correction [36, 37]. More exactly, we have divided the experimental modulus from BaBar, KLOE and CMD-2 experiments by the factor $\left|F_{\omega}(t)\right|$, where

$$
F_{\omega}(t)=\left(1+\epsilon \frac{t}{t_{\omega}-t}\right), \quad t_{\omega}=\left(M_{\omega}-i \Gamma_{\omega} / 2\right)^{2},
$$

with $M_{\omega}=0.7826 \mathrm{GeV}, \Gamma_{\omega}=0.0085 \mathrm{GeV}$ and $\epsilon=1.9 \times$ $10^{-3}[36,37$. 3 .

An important remark is that, except for the normalization condition (19) which is exact, all the inputs that we use are known only with some uncertainty. Following the discussion given in 22], in our analysis we have varied all the inputs simultaneously within their error intervals, taking the most conservative bounds on the quantity of interest consistent with the input, i.e. the largest upper bound and the smallest lower bound from the set of values obtained with specific inputs. In other words, we take the union of the allowed domains, obtained with specific input values inside the error intervals, for the quantity of interest. The procedure has been carried out efficiently with a combination of Mathematica and Fortran programs.

\section{Extraction of results}

\section{$4.1\left\langle r_{\pi}^{2}\right\rangle$ analysis}

We study first the impact of the conditions (2), (3), (19), (20) and (21) used as input in our formalism on the charge radius $\left\langle r_{\pi}^{2}\right\rangle$. To this end we calculate upper and lower bounds on the first derivative appearing in the expansion (1), by applying the general inequality (13) in the particular case $K=2$ and $N=2$. More precisely, we use as input, besides the normalization (19), one of the spacelike values (20) and a single experimental modulus $\left|F\left(t_{n}\right)\right|$ at an energy squared $t_{n}$ below the $\omega \pi$ threshold, from the sets reported in [6]-[11]. The errors on the phase, the spacelike value and the modulus were taken into account as explained at the end of Sec. 3 .

In Figs. 1 and 2 we present upper and lower bounds on $\left\langle r_{\pi}^{2}\right\rangle$ as functions of the energy $\sqrt{t}$ corresponding to the input modulus, for the input phase from [14] and [16], respectively. We have used input data from BaBar [6], KLOE [7, 8], CMD-2 [9, 10] and Belle [11] along the whole elastic region from the threshold $4 M_{\pi}^{2}$ to the first inelastic threshold $t_{\text {in }}$. For convenience we have connected the individual points by continuous lines 1 We show the

\footnotetext{
1 The number of experimental points below the $\omega \pi$ threshold reported by the BaBar experiment [6] is 221, while KLOE 7, 8 measured the modulus at 121 energies below $t_{\text {in }}$, CMD-2 [9, 10 ] at 34 and Belle 11] at 15 energies.
}

results obtained with the input from the first spacelike point $t=-1.60 \mathrm{GeV}^{2}$. The second spacelike point from (20) gives similar results.

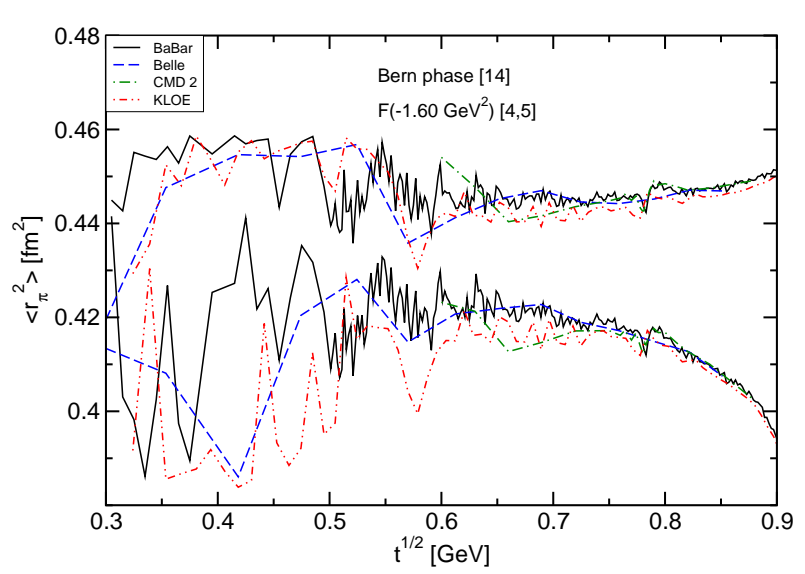

Fig. 1. Upper and lower bounds on $\left\langle r_{\pi}^{2}\right\rangle$ using as input one modulus value below the $\omega \pi$ inelastic threshold from the experiments BaBar, Belle, CMD-2 and KLOE, as functions of the energy $\sqrt{t}$ where the modulus was implemented. We used the Bern phase shift from 14] and the spacelike value $F\left(-1.60 \mathrm{GeV}^{2}\right)$ given in (20).

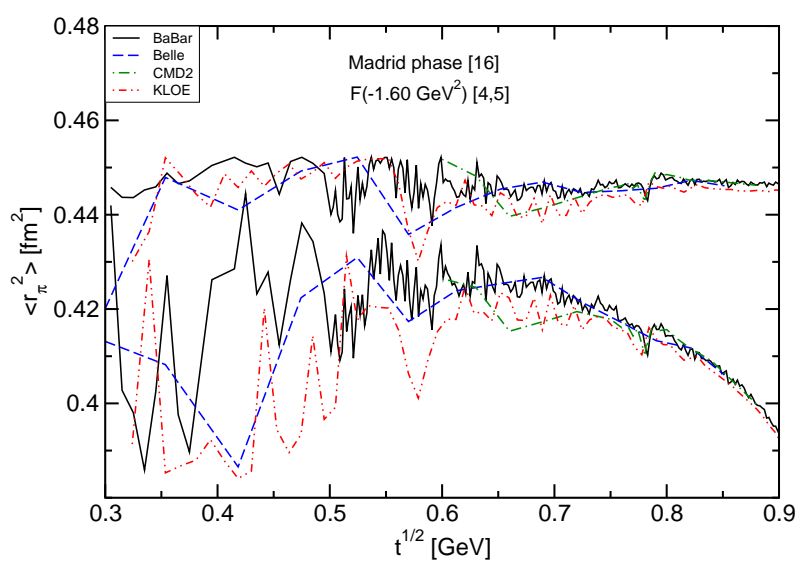

Fig. 2. As in Fig. [1 using as input the Madrid phase from [16].

The curves in Figs. 1 and 2 are not smooth: the bounds obtained for $\left\langle r_{\pi}^{2}\right\rangle$ vary quite drastically from point to point, especially at low energies, which means that they depend strongly on the experimental modulus used as input. In addition, there are several experimental data for which we do not even obtain real bounds for $\left\langle r_{\pi}^{2}\right\rangle$ : the quadratic equations derived from the inequality (13) have complex solutions for all the input values inside the error bars. In these cases (not shown in Figs. 1 and 2) the input modulus does not satisfy the analyticity constraints with the phase and the spacelike values used as input. Such inconsistencies in the data on modulus were noted already in our prior analysis [22]. 
The upper and lower bounds shown in Figs. 1 and 2 define, for each input modulus at a fixed energy, an allowed interval for the charge radius $\left\langle r_{\pi}^{2}\right\rangle$, which turns out to be quite narrow for some particular energies. In principle, if the data were consistent among each other, the final allowed domain for $\left\langle r_{\pi}^{2}\right\rangle$ would be given by the intersection of the allowed ranges obtained with particular inputs at fixed energies. The range defined by this intersection will be limited by the smallest upper bound and the largest lower bound shown in the figure. Of course, if the smallest upper bound turns out to be smaller than the largest lower bound the intersection is empty. In practice this turns out to be the case, if we consider all the points shown in Figs. 1 and 2, This is seen in the left part of Table 1, where $\left\langle r_{\pi}^{2}\right\rangle_{\max }$ is the lowest upper bound and $\left\langle r_{\pi}^{2}\right\rangle_{\min }$ is the largest lower bound upon all the experimental points below the $\omega \pi$ threshold. The corresponding values for the four experiments are indicated separately. For all experiments $\left\langle r_{\pi}^{2}\right\rangle_{\max }\left\langle\left\langle r_{\pi}^{2}\right\rangle_{\min }\right.$, which clearly indicates that there are inconsistencies between the measurements of the modulus at different energies, if we consider all the data between the elastic threshold $t_{+}$and the inelastic threshold $t_{\text {in }}$. The extraction of $\left\langle r_{\pi}^{2}\right\rangle$ is possible only if we restrict the intersection to a smaller energy interval.

It is obvious that the low energy region is questionable. The fairly drastic variations of the bounds obtained with the input from these energies may be explained by the big fluctuations of the data in this region and their mutual inconsistencies in spite of the large errors. Therefore, the data on modulus from the low energy region do not allow a consistent extraction of the radius.

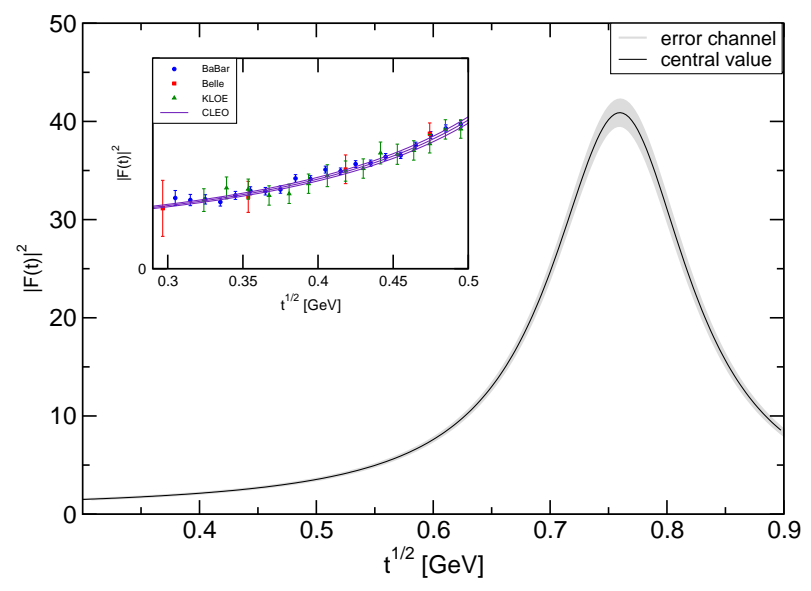

Fig. 3. Gounaris-Sakurai parametrization of the CLEO data 35] on the form factor modulus. The inset shows the parametrization together with the recent data below $0.5 \mathrm{GeV}$.

In order to test our formalism with a smoother input, we have considered the Gounaris-Sakurai (GS) based parametrization of the CLEO data on the modulus [35], shown in Fig. 3. In fact, the fit adopted in [35] does not satisfy all the constraints that the form factor must obey (in particular, as shown in [35], the normalization of the

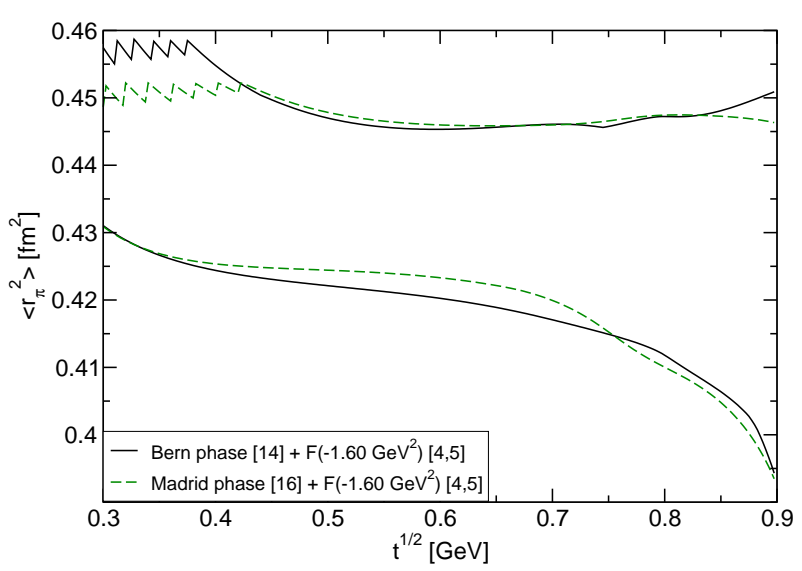

Fig. 4. Bounds on the charge radius using as input the modulus from the Gounaris-Sakurai parametrization of the CLEO data [35].

fit is allowed to float, and the resulting best fit explicitly violates the condition (19), being slightly larger than unity at $t=0)$. We note also that only data above $0.5 \mathrm{GeV}$ were included in the fit performed in [35], so the curve shown in Fig. 3 at low energies is actually an extrapolation. In our analysis we use the parametrization only to generate input on the unitarity cut, and impose the normalization condition on the admissible class of functions used in the derivation of the bounds.

To simulate the experimental situation, we have generated the modulus at 121 discrete points, using them and the corresponding errors 2 as input in our formalism. The upper and lower bounds on $\left\langle r_{\pi}^{2}\right\rangle$ obtained in this way for the two phases [14] and [16] are shown in Fig. 4 as functions of the energy at which the input modulus was used. One may see that the bounds depend now smoothly on the input energy, except for a few very small fluctuations at low energies, which may be explained by the fact that the simulated data do not satisfy the normalization condition $F(0)=1$ imposed in the formalism. The normalization chosen in [35], which artificialy increases the input modulus at low energies, can explain also the higher values of both the upper and lower bounds at the left edge of the figure. However, for energies above $0.5 \mathrm{GeV}$ where the fit is based on experimental data, the bounds are consistent with those given in Figs. 1 and 2, except for the fact that the curves are now smooth. This indicates that our formalism is sensitive to the noise in the input, as seen in Figs. 1 and 2, where we have not resorted to parametrizations of the data.

Taking into account the discussion above, for the real data we restrict our calculations to the input from energies above $0.65 \mathrm{GeV}$, which is free of the big fluctuations observed at low energies. We note that the bounds show a

\footnotetext{
${ }^{2}$ For simplicity, we assumed that the errors of the parameters are not correlated.
} 
Table 1. Intersections of the ranges of $\left\langle r_{\pi}^{2}\right\rangle$ obtained with various inputs.

\begin{tabular}{|c|c|c|c|c|c|c|c|c|c|}
\hline \multirow{3}{*}{ Spacelike data } & \multirow{3}{*}{$|F(t)|$} & \multicolumn{4}{|c|}{ Bern phase [14] } & \multicolumn{4}{c|}{ Madrid phase [16] } \\
\cline { 3 - 10 } & & All points included & \multicolumn{2}{|c|}{$(0.65-0.70) \mathrm{GeV}$} & \multicolumn{2}{|c|}{ All points included } & \multicolumn{2}{|c|}{$(0.65-0.70) \mathrm{GeV}$} \\
\cline { 3 - 10 } & & $\left\langle r_{\pi}^{2}\right\rangle_{\min }$ & $\left\langle r_{\pi}^{2}\right\rangle_{\max }$ & $\left\langle r_{\pi}^{2}\right\rangle_{\min }$ & $\left\langle r_{\pi}^{2}\right\rangle_{\max }$ & $\left\langle r_{\pi}^{2}\right\rangle_{\min }$ & $\left\langle r_{\pi}^{2}\right\rangle_{\max }$ & $\left\langle r_{\pi}^{2}\right\rangle_{\min }$ & $\left\langle r_{\pi}^{2}\right\rangle_{\max }$ \\
\hline \multirow{4}{*}{$F\left(-1.60 \mathrm{GeV}^{2}\right)$} & Belle & 0.4281 & 0.4177 & 0.4227 & 0.4446 & 0.4309 & 0.4184 & 0.4266 & 0.4447 \\
\cline { 2 - 10 } & BaBar & 0.4415 & 0.4358 & 0.4234 & 0.4431 & 0.4444 & 0.4361 & 0.4295 & 0.4430 \\
\cline { 2 - 10 } & CMD2 & 0.4232 & 0.4404 & 0.4171 & 0.4404 & 0.4264 & 0.4396 & 0.4194 & 0.4396 \\
\cline { 2 - 10 } & KLOE & 0.4304 & 0.4293 & 0.4201 & 0.4394 & 0.4312 & 0.4300 & 0.4235 & 0.4381 \\
\hline \hline \multirow{5}{*}{$F\left(-2.45 \mathrm{GeV}^{2}\right)$} & Belle & 0.4283 & 0.4173 & 0.4225 & 0.4436 & 0.4314 & 0.4179 & 0.4271 & 0.4437 \\
\cline { 2 - 10 } & BaBar & 0.4418 & 0.4349 & 0.4232 & 0.4421 & 0.4444 & 0.4351 & 0.4301 & 0.4421 \\
\cline { 2 - 10 } & CMD2 & 0.4230 & 0.4391 & 0.4161 & 0.4391 & 0.4267 & 0.4385 & 0.4193 & 0.4385 \\
\cline { 2 - 10 } & KLOE & 0.4305 & 0.4289 & 0.4196 & 0.4381 & 0.4317 & 0.4288 & 0.4235 & 0.4368 \\
\hline
\end{tabular}

Table 2. Weighted average of upper and lower bounds for $\left\langle r_{\pi}^{2}\right\rangle$ obtained with various inputs.

\begin{tabular}{|c|c|c|c|c|c|}
\hline \multirow{2}{*}{ Spacelike data } & $|F(t)|$ & \multicolumn{2}{|c|}{ Bern phase [14] } & \multicolumn{2}{c|}{ Madrid phase [16] } \\
\cline { 3 - 6 } & & $\left\langle r_{\pi}^{2}\right\rangle_{\min }$ & $\left\langle r_{\pi}^{2}\right\rangle_{\max }$ & $\left\langle r_{\pi}^{2}\right\rangle_{\min }$ & $\left\langle r_{\pi}^{2}\right\rangle_{\max }$ \\
\hline \multirow{4}{*}{$F\left(-1.60 \mathrm{GeV}^{2}\right)$} & Belle & 0.4152 & 0.4455 & 0.4162 & 0.4437 \\
\cline { 2 - 6 } & BaBar & 0.4172 & 0.4470 & 0.4187 & 0.4464 \\
\cline { 2 - 6 } & CMD2 & 0.4136 & 0.4457 & 0.4125 & 0.4457 \\
\cline { 2 - 6 } & KLOE & 0.4045 & 0.4467 & 0.4041 & 0.4446 \\
\hline \hline \multirow{5}{*}{$F\left(-2.45 \mathrm{GeV}^{2}\right)$} & Belle & 0.4136 & 0.4432 & 0.4151 & 0.4426 \\
\cline { 2 - 6 } & BaBar & 0.4159 & 0.4463 & 0.4180 & 0.4455 \\
\cline { 2 - 6 } & CMD2 & 0.4117 & 0.4447 & 0.4106 & 0.4447 \\
\cline { 2 - 6 } & KLOE & 0.4005 & 0.4456 & 0.4005 & 0.4434 \\
\hline
\end{tabular}

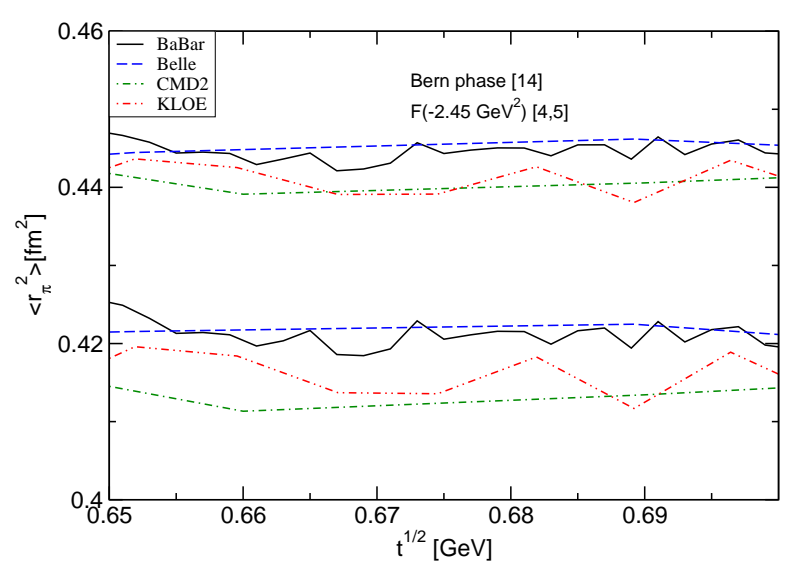

Fig. 5. Upper and lower bounds on $\left\langle r_{\pi}^{2}\right\rangle$ as a function of the energy in the region of stability where the modulus was implemented.

remarkable stability in the region $(0.65-0.70) \mathrm{GeV}$, and become weaker for the input from energies above $0.70 \mathrm{GeV}$. The upper and lower bounds on $\left\langle r_{\pi}^{2}\right\rangle$ obtained with various data sets in the region of stability as functions of the input energy are compared in Figs. 4 and 5 . We show for illustration the bounds obtained with the second spacelike point from (20), which are slightly better than those obtained with the first point due to its smaller relative error.

One can notice that the bounds obtained with the Madrid phase [16] are slightly shifted upwards compared to those obtained with the Bern phase [14]. We also see

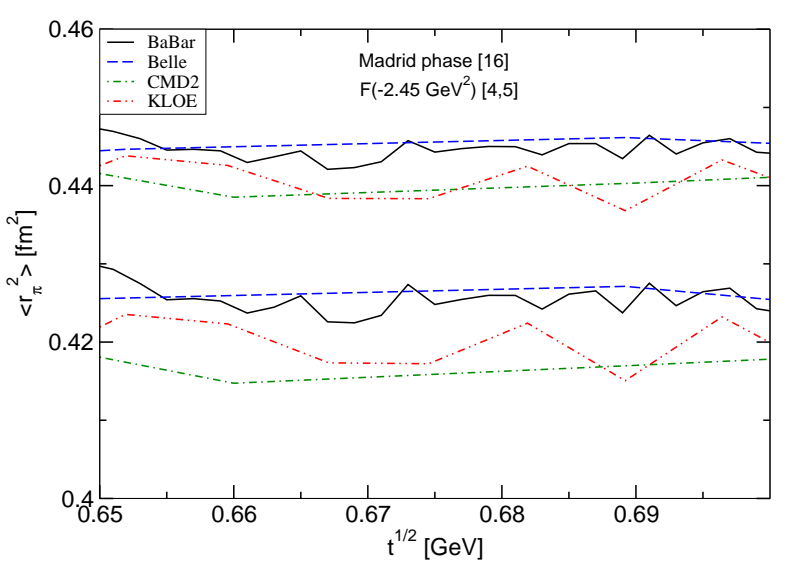

Fig. 6. As in Fig. 5, using the input phase from [16].

that the bounds from BaBar [6] and Belle [11] are shifted upwards compared to the bounds obtained with the data of CMD-2 [10] and KLOE [7, 8].

If we restrict the input to energies above $0.65 \mathrm{GeV}$, we obtain a nonzero intersection of the allowed ranges for the charge radius obtained with individual data on the modulus. Actually, the energies above $0.7 \mathrm{GeV}$ lead to weaker bounds and do not influence the intersection. So, we can use in the numerical calculations only the stable region (0.65-0.70) GeV. We show the results of the intersection in Table 1 where, for each data set, $\left\langle r_{\pi}^{2}\right\rangle_{\max }$ is the lowest upper bound and $\left\langle r_{\pi}^{2}\right\rangle_{\min }$ is the largest lower bound when the energy of the input modulus is varied along the stable region. From the values listed in the Table for all data sets 
we infer that the final range obtained by the intersection of the particular ranges is given by

$$
\left\langle r_{\pi}^{2}\right\rangle_{\min } \approx 0.42 \mathrm{fm}^{2}, \quad\left\langle r_{\pi}^{2}\right\rangle_{\max } \approx 0.44 \mathrm{fm}^{2} .
$$

Since a strict intersection of the $\left\langle r_{\pi}^{2}\right\rangle$ ranges upon the whole energy region does not exist, we may adopt a weaker definition of the final allowed range, by taking the weighted average of the upper (lower) bounds on $\left\langle r_{\pi}^{2}\right\rangle$ shown in Fig. 1. It is reasonable to use as weights in the average the experimental errors $\epsilon_{n}$ on the measured modulus $F_{n}$ as defined in (21). Thus we calculate for both the upper and lower bounds the weighted averages

$$
\left\langle r_{\pi}^{2}\right\rangle_{a v}=\frac{\sum_{n} w_{n}\left\langle r_{\pi}^{2}\right\rangle_{n}}{\sum_{n} w_{n}}, \quad w_{n}=1 / \epsilon_{n}^{2},
$$

where the sum runs over the individual energy points $t_{n}$. This definition suppresses the contribution of the lowenergy data, which have larger errors. The results are presented for all data sets in Table 2 from which we derive the weaker bounds

$$
\left\langle r_{\pi}^{2}\right\rangle_{\min , \mathrm{av}} \approx 0.40 \mathrm{fm}^{2}, \quad\left\langle r_{\pi}^{2}\right\rangle_{\max , \mathrm{av}} \approx 0.45 \mathrm{fm}^{2} .
$$

The range defined by the limits given in (23) and the more conservative limits (25) are our parametrization-free determinations based on the recent data on the modulus.

\section{$4.2 c-d$ analysis}

We now turn to the higher shape parameters $c$ and $d$ in the Taylor expansion (11). In our previous work [20] we have obtained an allowed domain in the $c$ - $d$ plane using as input the phase in the elastic region, the integral condition (3) and the experimental spacelike data given in (20). The modulus of the form factor in the timelike region below the inelastic threshold was not used as input in that study. Instead, we had used as input a range for the radius $\left\langle r_{\pi}^{2}\right\rangle$ taken from the literature. In the present work, we update the analysis by including as input the modulus measured below the inelastic threshold. It turns out that this input leads to strong constraints on the higher shape parameters at $t=0$ without any information on the charge radius.

The allowed domain in the $c$ - $d$ plane is obtained from the inequality (13) written in the particular case $K=$ 4, using as input the normalization (19) and one $(N=$ $1)$ or two $(N=2)$ additional values at interior points. One can see that the first derivative at $t=0$, i.e. the radius $\left\langle r_{\pi}^{2}\right\rangle$, appears in the coefficients of the quadratic relation for the parameters $c$ and $d$ resulting from the determinant (13). Moreover, the quadratic equation for $c$ at a fixed $d$, for instance, has real solutions only for $\left\langle r_{\pi}^{2}\right\rangle$ in the allowed range determined from the same inequality (13), as shown in the previous subsection. So, in principle it is not necessary to put a limitation on the range of the charge radius when deriving constraints on the higher shape parameters $c$ and $d$ : all we have to do is to vary the value of $\left\langle r_{\pi}^{2}\right\rangle$ over an interval large enough as to cover the allowed range, and take the weakest bounds obtained with this variable input.

However, if the modulus below $t_{\text {in }}$ is not included, the constraint on the radius is rather weak, and the corresponding domain in the $c$ - $d$ plane will be quite large. Therefore, in this case one has to adopt as input a certain smaller range for $\left\langle r_{\pi}^{2}\right\rangle$ in order to actually constrain the higher derivatives. In [20] we have taken as input the narrow range $\left\langle r_{\pi}^{2}\right\rangle=(0.435 \pm 0.005) \mathrm{fm}^{2}$, suggested by some previous works. We now repeated first that analysis, using for $\left\langle r_{\pi}^{2}\right\rangle$ the larger range $(0.42-0.44) \mathrm{fm}^{2}$, derived in Eq. (23).

The results are presented in Fig. 7 for the two input phases, Bern [14] and Madrid [16] and the spacelike value at $-2.45 \mathrm{GeV}^{2}$. We recall that for a fixed input the allowed domain is the interior of an ellipse in the $c$ - $d$ plane, whose boundary is given by the upper and lower bounds on $d$ at each fixed $c$, found by solving a quadratic equation. The errors on the phase and spacelike value were taken into account, as explained at the end of Sec. 3 , by varying the input quantities inside the error intervals and taking the weakest bounds, i.e. the union of the corresponding ranges of $d$ at each fixed $c$. The final allowed domain is no longer an ellipse, but has a more complicated shape.

Fig. 7 shows that the domains obtained are consistent with the ranges of $c$ and $d$ quoted in [20], being slightly weaker due to the more conservative input for the charge radius adopted now. Note that we use also a different weight function $\rho(t)$ in the condition (3): while in [20] we adopted a special weight relevant for the calculation of the muon's $(g-2)$, we now make calculations with $\rho(t)=1 / t$. In fact, we have tried also other weights of the form (44), and found that the constraints on the shape parameters at $t=0$ are not very sensitive to the choice of the weight.

We then include as input the value of the modulus $\left|F\left(t_{n}\right)\right|$ at a fixed energy $t_{n}$ below the inelastic threshold. In this analysis we used only BaBar data from the stability region defined above. As we mentioned, in this case it is not necessary to restrict the charge radius, because the allowed range imposed by the input is already quite narrow. In the numerical calculations, we restricted however $\left\langle r_{\pi}^{2}\right\rangle$ to the interval between $0.41 \mathrm{fm}^{2}$ and $0.45 \mathrm{fm}^{2}$, which covers practically all the ranges obtained at fixed energies with BaBar data. Extending this range would not have perceptible effects. At each energy, we varied also the input quantities (the phase, the spacelike datum and the modulus $\left.\left|F\left(t_{n}\right)\right|\right)$ within their error bars, and took the weakest bounds on $d$ at each fixed $c$. Finally, the input energy $t_{n}$ was varied in the stability region, and the intersection of the particular allowed domains was derived, by taking the smallest upper bound and the largest lower bound on $d$ at each admissible $c$. The boundary of the resulting allowed domain, which has a more complicated shape, was found numerically. In Fig. 8 we present the allowed domains in the $c$ - $d$ plane obtained by the above procedure. The improvement brought by the modulus information is considerable: the domains are now much smaller than those in 
Fig. 7 From these domains we predict the allowed ranges:

$$
\begin{aligned}
& c \in(3.79,4.00) \mathrm{GeV}^{-4}, \\
& d \in(10.14,10.56) \mathrm{GeV}^{-6},
\end{aligned}
$$

with a strong correlation between the two parameters.

We emphasize however that the ranges (26) are based only on the BaBar data and should be regarded as provisional. Indeed, the small inconsistencies beween the data sets, visible already in the extraction of the radius, are expected to be amplified in the higher derivatives. Therefore, precise experimental predictions for the higher shape parameters will be possible only when the discrepancies between the data sets will be understood and consistent data will be available.
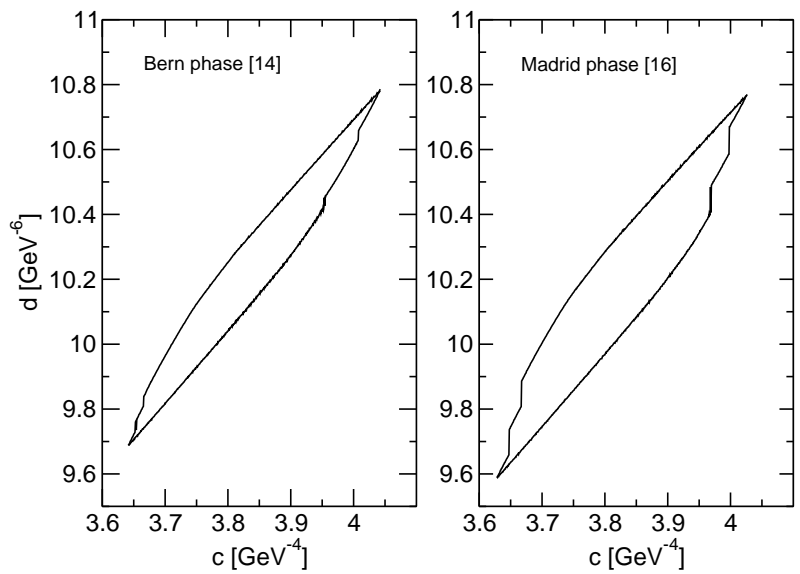

Fig. 7. Allowed domain in the $c-d$ plane obtained without timelike modulus data.
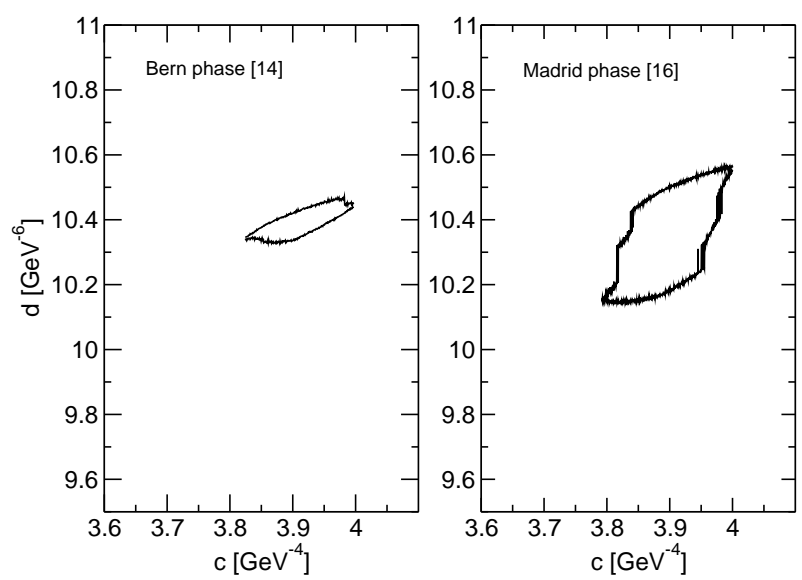

Fig. 8. Allowed domain in the $c-d$ plane obtained with modulus data from BaBar [6] in the energy region 0.65-0.70 GeV.

\section{Discussion and conclusions}

Our work was motivated by the fact that the phenomenological knowledge of the pion electromagnetic form factor, which improved considerably in recent years, comes from different sources: the phase below $0.917 \mathrm{GeV}$ is known via Fermi-Watson theorem from the $P$-wave phase shift of $\pi \pi$ scattering calculated from the Roy equations, the modulus has been measured recently by high statistics experiments on $e^{+} e^{-}$annihilation or $\tau$-decays, and measurements on the spacelike axis from electroproduction data with improved accuracy were also reported. Suitable tools based on analyticity must be devised for exploiting the available knowledge in order to make model-independent predictions in regions not directly accessible to experiment.

If the modulus and the phase are both known along an energy interval of the unitarity cut, the function can be reconstructed in principle everywhere, by the uniqueness of analytic continuation. However, since the input is known with some uncertainty, the reconstruction is practically impossible. Indeed, analytic continuation is unique, but it is also an unstable (ill-posed) problem in the Hadamard sense [17], which means that the small uncertainties along the original range may be amplified arbitrarily in much of the complex plane outside that range.

In our analysis we applied a formalism that exploits in an optimal way the information on the phase and modulus on the unitarity cut, leading to results independent on the unknown phase above the inelastic threshold. Combined with the well-known analytic interpolation theory, it allows us to include information or derive predictions on the values at points inside the analyticity domain. The formalism also acts as a sensitive devise for testing the consistency of the various experimental data sets with analyticity and among themselves. The technique has been already applied to the pion form factor in Refs. 20, 21, 22].

In the present work we studied the impact of the timelike modulus data below the inelastic threshold on the determination of the charge radius $\left\langle r_{\pi}^{2}\right\rangle$ and the higher shape parameters $c$ and $d$ in the Taylor expansion (1). To our knowledge, this is the first application of the highstatistics modulus measurements to the extraction of the pion charge radius and the higher shape parameters.

Using as input the conditions (2), (3), (19), (20) and (21), we calculated from the inequality (13) upper and lower bounds on the first derivative appearing in the expansion (1). The uncertainties of the phase in the elastic region, the spacelike value and the modulus were taken into account by varying the input inside the error intervals and taking the largest allowed range. The final prediction for $\left\langle r_{\pi}^{2}\right\rangle$ is given in principle by the intersection of all the ranges obtained with an input modulus at fixed energy below the inelastic threshold.

The analysis presented in Sec. 4 shows that, for all the experimental data sets, this intersection turns to be empty, which indicates that there are inconsistencies in the input data. Especially, the data at low energes lead to results that strongly fluctuate from point to point and are not compatible among them, in spite of the large experimental errors. However, if we restrict the input to mea- 
surements of the modulus at energies greater than 0.65 $\mathrm{GeV}$, the results from all the experiments, BaBar, KLOE, CMD-2 and Belle, and the two phases, Madrid and Bern, are consistent to a large extent and lead to the prediction (23), which we write as

$$
\left\langle r_{\pi}^{2}\right\rangle \in(0.42,0.44) \mathrm{fm}^{2}
$$

Our parametrization-free prediction (27) is consistent with most of the results based on specific parametrizations reported in the literature.

Note that we avoid the presentation of this result in terms of a central value and an error, for instance $\left\langle r_{\pi}^{2}\right\rangle=$ $(0.43 \pm 0.01) \mathrm{fm}^{2}$ instead of (27). The reason is that, although the probability of the parameters to be in the predicted allowed ranges follows from the probabilities of the error intervals inside which we varied the input quantities, we cannot actually attach precise probabilities to the specific values inside the allowed domains. Indeed, even for the central values of the input we obtain a range for the parameters of interest, not a definite prediction. As a statistical interpretation is difficult to give, we can assume that all the values in the predicted range are equally probable.

For the higher shape parameters $c$ and $d$ the ranges (26) obtained with the BaBar data are very stringent, illustrating the great constraining power of the method. We emphasize however that the $c-d$ domains are very sensitive to the input modulus. Therefore, the results (26) should be regarded as provisional until the discrepancies between the experimental data sets are understood and consistent data are available.

We recall that in 22] the mathematical formalism was applied in a somewhat opposite way, i.e. we adopted as input the value of the radius and derived bounds on the modulus below the $\omega \pi$ threshold. In fact, we have used as input precisely the range (27), which we now obtained from the information on the modulus, and found that the derived bounds are consistent with the experimental data at higher energies, but in some disagreement at low energies. Thus, the two analyses are perfectly consistent. In particular, as already mentioned, the bounds on the modulus at low energies, derived with the input range (27) for the radius, are more precise than the data. Therefore, they can be used for improving the evaluation of the two-pion contribution to the muon's magnetic anomaly, especially the contribution of the region below $0.5 \mathrm{GeV}$, which is small but is known to have a relatively large error. This problem will be studied in a future work.

Acknowledgement: IC acknowledges support from the Program Nucleu under Contract PN 09370102/2009, and from Program Idei-PCE, Contract No 121/2011. ISS acknowledges the support from Deutsche Forschungsgemeinschaft Research Unit FOR 1873 Quark Flavour Physics and Effective Theories, Contract No. KH 205/2-1.

\section{References}

1. S.R. Amendolia et al. [NA7 Collaboration], Nucl. Phys. B 277 (1986) 168.

2. C.N. Brown, C.R. Canizares, W.E. Cooper, A.M. Eisner, G.J. Feldmann, C.A. Lichtenstein, L. Litt, W. Loceretz, V.B. Montana and F.M. Pipkin Phys. Rev. D 8, 92 (1973).

3. C.J. Bebek et al.,' Phys. Rev. D17, 1693 (1978).

4. T. Horn et al. [Jefferson Lab F(pi)-2 Collaboration], Phys. Rev. Lett. 97 (2006) 192001 nucl-ex/0607005.

5. G. M. Huber et al. [Jefferson Lab Collaboration], Phys. Rev. C 78 (2008) 045203 arXiv:0809.3052 [nucl-ex]].

6. B. Aubert et al. [BABAR Collaboration], Phys. Rev. Lett. 103 (2009) 231801 arXiv:0908.3589 [hep-ex]].

7. F. Ambrosino et al. [KLOE Collaboration], Phys. Lett. B 670 (2009) 285 arXiv:0809.3950 [hep-ex]].

8. F. Ambrosino et al. [KLOE Collaboration], Phys. Lett. B 700 (2011) 102 arXiv:1006.5313 [hep-ex]].

9. R.R. Akhmetshin et al., [CMD-2 Collaboration], JETP Lett. 84 (2006) 413 [Pisma Zh. Eksp. Teor. Fiz. 84 (2006) 491] hep-ex/0610016.

10. R. R. Akhmetshin et al. [CMD-2 Collaboration], Phys. Lett. B 648 (2007) 28 [hep-ex/0610021.

11. M. Fujikawa et al. [Belle Collaboration], Phys. Rev. D 78 (2008) 072006 arXiv:0805.3773 [hep-ex]].

12. B. Ananthanarayan, G. Colangelo, J. Gasser and H. Leutwyler, Phys. Rept. 353 (2001) 207 hep-ph/0005297.

13. G. Colangelo, J. Gasser and H. Leutwyler, Nucl. Phys. B 603 (2001) 125 hep-ph/0103088.

14. I. Caprini, G. Colangelo and H. Leutwyler, Eur. Phys. J. C 72 (2012) 1860 arXiv:1111.7160 [hep$\mathrm{ph}]$.

15. R. Kaminski, J. R. Pelaez and F. J. Yndurain, Phys. Rev. D 77 (2008) 054015 arXiv:0710.1150 [hep-ph]].

16. R. Garcia-Martin, R. Kaminski, J. R. Pelaez, J. Ruiz de Elvira and F. J. Yndurain, Phys. Rev. D 83 (2011) 074004 arXiv:1102.2183 [hep-ph]].

17. S. Ciulli, C. Pomponiu and I. Sabba-Stefanescu, Phys. Rept. 17 (1975) 133.

18. G. Abbas, B. Ananthanarayan, I. Caprini, I. Sentitemsu Imsong and S. Ramanan, Eur. Phys. J. A 45 (2010) 389 arXiv:1004.4257 [hep-ph]].

19. I. Caprini, Eur. Phys. J. $\quad$ C 13 (2000) 471 [hep-ph/9907227.

20. B. Ananthanarayan, I. Caprini and I. S. Imsong, Phys. Rev. D 83 (2011) 096002 arXiv:1102.3299 [hep-ph]].

21. B. Ananthanarayan, I. Caprini and I.S. Imsong, Phys. Rev. D 85 (2012) 096006 arXiv:1203.5398 [hep-ph]].

22. B. Ananthanarayan, I. Caprini, D. Das and I. S. Imsong, Eur. Phys. J. C 72 (2012) 2192 arXiv:1209.0379 [hep-ph]].

23. G. Colangelo, M. Finkemeier and R. Urech, Phys. Rev. D 54, 4403 (1996) arXiv:hep-ph/9604279.

24. J. Bijnens, G. Colangelo and P. Talavera, JHEP 9805, 014 (1998) arXiv:hep-ph/9805389. 
25. J.Bijnens and P. Talavera, JHEP 0203, 046 (2002) arXiv:hep-ph/0203049.

26. P. Maris and P. C. Tandy, Phys. Rev. C 61 (2000) 045202 nucl-th/9910033.

27. S. Aoki et al. [JLQCD Collaboration and TWQCD Collaboration], Phys. Rev. D 80, 034508 (2009) arXiv:0905.2465 [hep-lat]].

28. J. F. de Troconiz and F. J. Yndurain, Phys. Rev. D 71, 073008 (2005) arXiv:hep-ph/0402285.

29. P. Masjuan, S. Peris and J. J. Sanz-Cillero, Phys. Rev. D 78, 074028 (2008) arXiv:0807.4893 [hep-ph]].

30. T. N. Truong, arXiv:hep-ph/9809476.

31. R. Barate et al. [ALEPH Collaboration], Z. Phys. C 76, 15 (1997).

32. G. J. Gounaris and J. J. Sakurai, Phys. Rev. Lett. 21, 244 (1968).

33. N.N. Meiman, Sov. Phys. JETP. 17 (1963) 830.

34. P. Duren, Theory of $H^{\mathrm{p}}$ Spaces, Academic Press, New York, 1970.

35. S. Anderson et al. [CLEO Collaboration], Phys. Rev. D 61 (2000) 112002 hep-ex/9910046.

36. H. Leutwyler, hep-ph/0212324

37. C. Hanhart, Phys. Lett. B715 (2012) 170, arXiv:1203.6839 [hep-ph]. 